\title{
Profile of Visceral Injuries in Blunt Trauma Abdomen in Jammu Region: A Hospital Based Study
}

\author{
Tariq P Azad ${ }^{1}$, Shyam Gupta ${ }^{2}$, Gopal Sharma ${ }^{3}$, Manmeet kaur ${ }^{4}$, \\ Sandeep Bhat 5 . \\ ${ }^{1}$ Professor, Department of Surgery, GMC Jammu \\ ${ }^{2}$ Lecturer, Department of Surgery, GMC Jammu \\ ${ }^{3}$ senior Resident Department of Surgery, GMC Jammu \\ ${ }^{4}$ Lecturer, Department of Surgery, GMC Jammu \\ ${ }^{5}$ Lecturer, Department of Surgery, GMC Jammu
}

\begin{abstract}
:
Context: Injury has been a constant companion of mankind since the times immemorial. Although the modern trauma care has scaled new heights vis-a vis the infrastructure and the technical expertise, trauma remains defiant and continues to pose a huge challenge to the modern society in view of ever increasing magnitude.

Material and methods: A series of 100 patients admitted with blunt trauma abdomen over a period of one year in Government Medical College, Jammu was enrolled in the study design.

Result-Abdominal trauma usually affects the younger generation, in particular the male gender. Majority of the patients were victims of road traffic accidents. The overall mortality from blunt trauma abdomen was $11 \%$.

Conclusion: No age is exempted from abdominal trauma but a high index of suspicion, early diagnosis and requisite management are very important for a favorable outcome.
\end{abstract}

Keywords: Blunt trauma, Abdomen, Visceral injury, Accident

\section{Introduction}

Abdominal injuries are seen in 2 to 5 percent of all accidents and are the leading injury in $51.6 \%$ of fatal accidents ${ }^{1}$. Trauma is the leading cause of death in persons under 45 years of age, $10 \%$ of these fatalities are attributable to abdominal injuries ${ }^{2}$. There has been 10 fold increase in terrorist acts around the world over the last two decades so it is of utmost importance for medical personal to be well versed with the management of such victims ${ }^{3}$. Abdominal visceral injury is one of the commonest entities dealt by a general surgeon throughout his career. Diagnosis of visceral injuries following blunt trauma abdomen is relatively difficult in comparison to the penetrating injuries which is quiet conspicuous. It presents even greater diagnostic challenge when the sign and symptoms are not so obvious either because of early presentation or due to associated conditions like head injury, alcohol and drug abuse. Arguably so, the mortality remains relatively high and hence merits a thorough and an urgent evaluation in order to prevent life threatening complications and improve survival. Pre- hospital transportation, initial thorough assessment, resuscitative measures and correct diagnosis are of paramount importance. Proper investigations and repeated examination guide regarding the line of management, be it conservative or surgical. The study was undertaken in Government Medical College, Jammu to describe the epidemiology of visceral injuries in patients presenting with blunt trauma abdomen and to evaluate the pattern of visceral injuries.

\section{Material and methods}

This prospective study was carried out in patients admitted with blunt trauma abdomen in Government Medical College Hospital over a period of one year. Patients were evaluated as under-

1. History: A brief and relevant history was obtained. History suggestive of any co-morbidity and substance abuse was noted.

2. Physical examination: A quick and thorough examination was conducted. Hemodynamic parameters, clinical pallor and level of consciousness were evaluated in particular. Systemic examination was conducted were ever feasible.

3. Local examination: Abdomen was examined for any tell tale signs of trauma such as bruises, abrasions, tenderness, rebound tenderness, guarding etc. Patients with hemodynamic instability were subjected to urgent resuscitative measures as per the protocol. In patients, who presented with hypovolumeic shock at the time of admission, all measures to manage shock in the form of crystalloids, colloids and blood transfusions were given priority over any further diagnostic procedure. 
4. Laboratory tests: Blood sample was obtained for requisite investigations in particular, the blood grouping and the haemogram, rests of the investigations were ordered as per the clinical profile of the patient.

5. X -ray abdomen/ chest standing AP view was taken to look for any air under diaphragm, fracture of lower ribs, spine and pelvis, loss of psoas outline, ground glass appearance.

6. Ultrasound abdomen was done in all patients to look for any free fluid / air and or visceral injury. FAST scan was obtained in the emergency room itself in critical patients who were haemodynamically unstable. Free fluid was tapped under guidance for examination.

7. CECT Abdomen was obtained in haemodynamically stable patients with ultrasound abdomen findings suggestive of solid organ injury and or free fluid in peritoneal cavity. Patients with signs of peritonism with inconclusive ultrasound / $\mathrm{x}$-ray findings were also subjected to CECT. $5-7 \mathrm{~mm}$ thick slices were taken with spiral CT scanner (CTi)/multi-slice somatom spirt.50-100cc of non-ionic contrast was used.

8. Exploratory laparotomy was performed in following patients:

- Perforation peritonitis

- Haemoperitoneum with peritonism.

- Evidence of significant visceral/ solid organ injury.

- Positive paracentesis in an unstable patient.

Intraoperative findings were noted and negative laparotomies reported.

9. Conservative management: Rest all patients were managed conservatively as per established protocol.

\section{Results}

Most common age group involved in our study was between 21 to 30 years of age (32\%) and only 3 patients were above 60 years. Males accounted for more than two third of cases $(81 \%)$ (Table No. 1). Majority of the patients were the victims of road traffic accidents $(53 \%)$, followed by fall $(31 \%)$ and interpersonal violence (4\%). $30 \%$ sustained injury at home and $11 \%$ at work place. Road traffic accidents affected all age groups but majority of patients presenting with fall belonged to extreme age groups. Out of 52 patients who sustained roadside injuries, 45 were males. Most of patients reported within 6 hours of injury (62\%). Five patients took more than 24 hours to reach hospital usually due to long distance they had to travel. Majority of patients presented with pain abdomen (88\%) followed by vomiting (20\%). Only few patients were brought in an unconscious state (5\%). Tenderness was the most common abdominal sign (83\%) followed by guarding (33\%) and distension (26). More than one sign were present in some of the patients. External injuries over abdomen like bruise / abrasion were present only in $11 \%$ of patients. Associated extra- abdominal injuries were present in a significant number of patients (42\%), most common being injuries to limbs including pelvis (22\%), followed by injury to chest (11\%) and head (9\%). Peritoneal tap was positive in $43 \%$ of patients with intra abdominal injuries. A positive paracentesis is always significant, whereas a negative paracentesis doesn't exclude an internal injury.USG abdomen was done in 88 patients and haemoperitoneum was detected in 77 patients and visceral injuries were detected in 40 patients. CECT abdomen was done in 22 haemodynamically stable patients and visceral injuries were detected in all of them. During this study majority of the patients were explored (71\%). At laparotomy, spleen $(43.60 \%)$ was the most common viscera to be injured followed by liver $(30.9 \%)$, 11 patients had multiple visceral injuries (Table No. 2). Most injuries were of grade 3 in spleen (39\%) and grade 2 in liver (46\%) (Table No. 3). Splenectomy and hepatorraphy was done in patients where ever indicated as per the institutional protocol. All small gut and a single gastric perforation were closed primarily and cholecystectomy was done in 2 cases of gall bladder perforation. The overall mortality from blunt trauma abdomen was $11 \%$. Postoperative complications in the form of wound infection and sepsis were noted in five patients.

\section{Discussion}

Trauma represents a major public health problem and affects all sections of the society irrespective of the socioeconomic status. With increasing incidence of road traffic accidents, industrial events, and interpersonal violence, the burden is ever increasing.

In our study the most common age group involved was between 21-30 years accounting for $32 \%$ of all cases. Similar results were reported by Lone $\mathrm{G} \mathrm{N}$ et $\mathrm{al}^{3}$, Deodhar S D et $\mathrm{al}^{4}$ and Mohapatra S et $\mathrm{al}^{5}$ with respective findings of $35.41 \%, 70.6 \%, 33.3 \%$. In the review conducted by Cochran et $\mathrm{al}^{6}, 33 \%$ of the patients were in early twenties. This is most probably due to active and productive nature of the group. Males constituted about $81 \%$ of the cases in our study and $68.7 \%$ of the patients in another study ${ }^{6}$. The male: female ratio in our study was 4:1. The male: female ratio was $3: 1$ in the studies conducted by Lone $\mathrm{G} \mathrm{N}$ et $\mathrm{al}^{3}$ and Udeoyop U W et $\mathrm{al}^{7}$. Predominance of the male gender is an outcome of the outdoor activities, involvement in frequent travel and behavioral pattern exhibited by young adult men. Major mechanism of injury was road traffic accident accounting for $53 \%$ of cases. Road traffic accidents were responsible for $62 \%^{5}, 50 \%^{7}, 64.9 \%^{8}$ cases of blunt 
trauma abdomen in other similar studies.Preventive measures to reduce the incidence of road traffic accidents can significantly reduce the burden of trauma. Requisite infrastructure, strict enforcement of traffic rules and caution while driving hold the key. In our study five patients reached the hospital after 24 hours out of which 2 patients died thereby stressing the need for timely management and exposing the pit falls of trauma care delivery. Most of the patients presented with pain abdomen (88\%), followed by vomiting (20\%), while 5\% were brought in an unconscious state. Alcohol intoxication was observed in $6 \%$ of patients. In our study abdominal tenderness was present in $83 \%$ of patients and guarding in $33 \%$ which is comparable to the studies conducted by Mohapatra S et $\mathrm{al}^{5}$. Although bowel sounds were absent in $24 \%$ of patients it is not considered as a reliable criteria so far as the assessment of underlying visceral injury is considered ${ }^{9,10}$. In our study associated extra abdominal injury was noted in $43 \%$ of patients, most common being injury to lower extremity including pelvis ( $22 \%)$ followed by chest $(11 \%)$ and head injury $(9 \%)$ which is similar to the studies conducted by Shaftan G W et $\mathrm{al}^{9}$, Williams R D et $\mathrm{al}^{11}$. However the study conducted by Chalya $\mathrm{P} \mathrm{L} \mathrm{et} \mathrm{al}{ }^{8}$ reported an incidence to be 96.8\%. The mortality in our study included two patients with severe head injury, one patient with associated pelvis trauma and another patient with alcohol intoxication with polytrauma. Concurrent extra abdominal injury and/ or altered sensorium due to head injury or alcohol intoxication further compound the problem. This is in agreement with studies conducted by Mohapatra $\mathrm{S}$ et $\mathrm{al}^{5}$. McAnena $\mathrm{O} \mathrm{J}$ et al ${ }^{12}$ and calls for thorough evaluation and management of polytrauma patients with a well organized trauma care team in order to reduce the mortality in such cases. CECT abdomen was performed in 22 haemodynamically stable patients. CT scan had the highest sensitivity for detecting the injuries of liver $(100 \%)$ and spleen $(86.6 \%)$. The specificity of the method for detecting retroperitoneal hematoma $(100 \%)$ and injuries to kidney $(93.5 \%)$ was higher than other organs.

The accuracy of CT images to detect the injuries to spleen, liver, kidney and retroperitoneal hematoma has been reported to be $96.1 \%, 94.4 \%, 91.6 \%$ and $91.6 \%$ respectively ${ }^{13}$. The sensitivity of CT in the diagnosis of small intestine injury has been reported to be as low as $75 \%$ o and $64 \%$ for bowel or mesenteric injury therefore serial abdominal examination is of high importance ${ }^{14,15}$. CT is still considered the "modality of choice" for diagnosing small bowel perforation because of its high sensitivity to extra luminal air (100\% sensitivity when CT was obtained more than 8 hours after injury). Injury to spleen was noted in 8 patients, kidney in 5; liver, urinary bladder injury and retroperitoneal hemorrhage were noted in 3 patients each. All were managed conservatively except for a child of polytrauma with grade 2 splenic injuries with persistent tachycardia and tenderness, who was explored and splenectomy was done. In our study 71 patients were explored and among these splenic injuries was present in 31 , liver injury in 22 , bowel injury in 13 , mesenteric tear in 4 , retroperitoneal hematoma in 4, gall bladder perforations in 2, renal and urinary bladder in 1 patient each. Eleven patients had injury to more than one viscus. In another study by Perry $\mathrm{JF}^{16}$ almost similar incidence of visceral injuries at laparotomy viz; spleen in $44 \%$, liver in $22 \%$, gut in $12 \%$, mesentery in $12 \%$ and kidney in $3 \%$ patients was noted. Similarly in the study conducted by Everard $\mathrm{FC}^{17}$, spleen was reported to be injured in $42.18 \%$, followed by liver in $35.63 \%$, retroperitoneal hematoma was present in $14.59 \%$, mesenteric tear in $13 \%$, diaphragmatic injury in $5.28 \%$, bowel injury in $4.71 \%$, genitourinary injury in $6.89 \%$, vascular injury in $3.10 \%$, others in $0.05 \%$. In our study Spleen was the most common organ injured on exploratory laprotomy. Similarly, Spleen was found to be the most commonly injured organ on exploratory laparotomy in the studies conducted by Masau $\mathrm{P}$ et a ${ }^{18}$ and Nyongole $\mathrm{OV}$ et al ${ }^{19}$. A missed splenic injury is the most common cause of preventable death in blunt trauma patients thereby calling for an early diagnosis, timely and adequate resuscitation and urgent surgical intervention for favorable outcome ${ }^{20}$.

\section{Conclusion}

Blunt trauma abdomen continues to pose a challenge to the medical fraternity in particular and society at large. Reforms in the trauma care delivery and targeted preventive measures to reduce the burden of trauma especially due to road traffic accidents in the society constitute a pragmatic approach towards the appropriate management of the problem.

\section{Acknowledgement}

The authors are grateful to Dr. Ghansnyam Dev, M.D. (Radiodiagnosis), Professor \& Head, Post Graduate Department of Radiodiagnosis and Imaging, Government Medical College and Hospital, Jammu for his valuable advice and support in completion of this study.We are also highly grateful and thankful to DR SONAM STOBGAIS who contributed a lot to this work. Infact without him it would not have been possible but he left us at a very young age. We all miss him.May his soul rest in peace.

Table 1: Age and sex distribution

\begin{tabular}{|c|c|c|c|}
\hline Age in years & Male & Female & Total \\
\hline $0-10$ & 10 & 03 & 13 \\
\hline $11-20$ & 14 & 05 & 19 \\
\hline
\end{tabular}


Profile Of Visceral Injuries In Blunt Trauma Abdomen In Jammu Region: A Hospital .

\begin{tabular}{|c|c|c|c|}
\hline $21-30$ & 30 & 02 & 32 \\
\hline $31-40$ & 14 & 04 & 18 \\
\hline $41-50$ & 08 & 03 & 11 \\
\hline $51-60$ & 04 & 00 & 04 \\
\hline $61-70$ & 00 & 00 & 00 \\
\hline $71-80$ & 01 & 02 & 03 \\
\hline Total & 81 & 19 & 100 \\
\hline
\end{tabular}

Table 2: Laparotomy findings

\begin{tabular}{|l|c|c|}
\hline \multicolumn{1}{|c|}{ Visceral injury } & No. of patients & \%age \\
\hline Spleen & 31 & 43.60 \\
\hline Liver & 22 & 30.90 \\
\hline Jejunal perforation & 06 & 8.45 \\
\hline Retroperitoneal haematoma & 06 & 8.45 \\
\hline Ileal perforation & 05 & 7.04 \\
\hline Mesenteric tear & 04 & 5.60 \\
\hline Large gut injury & 02 & 2.80 \\
\hline Gastric perforation & 02 & 2.80 \\
\hline GB perforation & 02 & 2.80 \\
\hline Kidney & 01 & 1.40 \\
\hline Bladder & 01 & 1.40 \\
\hline
\end{tabular}

Table 3: Grades of injury

\begin{tabular}{|l|c|c|}
\hline \multicolumn{1}{|c|}{ Grading } & No. of patients with liver injury. & No. of patients with splenic injury. \\
\hline Grade 1 & 4 & 3 \\
\hline Grade 2 & 10 & 10 \\
\hline Grade 3 & 8 & 12 \\
\hline Grade 4 & 0 & 6 \\
\hline Grade 5 & 0 & 0 \\
\hline
\end{tabular}

\section{References}

[1]. Probst M. Early treatment of abdominal injury. Langenbecks Arch Chir, Suppl., Kongressbd, 1991: 84-87

[2]. 2 Kumar M M, Venkataramanappa M, Venkataratnam I., Kumar N V., Babji K. Prospective evaluation of blunt abdominal trauma by computed tomography. Indian J Radiol Imaging 2005; 15:167-73

[3]. Lone G N, Peer G Q, Warn K A. et al. An experience with abdominal trauma in adults in Kashmir. J K Practitioner 2001; 8:225230.

[4]. Deodhar S D, Patel N P, Shah K B. et al. Blunt and penetrating abdominal injuries- a study of 51 cases. JPGM. 1983; 29:96-99.

[5]. Mohapatra S., Pattanayak S P., Rao KRRM., Bastia B. Options in the management of solid visceral injuries from blunt abdominal trauma. Indian J Surg 2003;65:263-8.

[6]. Cochran W, Sobat W S. Open vs closed diagnostic peritoneal lavage, a multiphasic prospective randomized comparison. Ann. Surg. 1984;200:24-28.

[7]. Udeoyop U W. and Iwatt A R. Abdominal trauma in South East Nigeria. Cent. Afr. J. Med. 1991;37:409-415

[8]. Chalya P L. and Mabula J B. Abdominal trauma experience over a two year period at a tertiary hospital in north western Tanzania: a prospective study of 396 cases. Tanzania Journal of Health Research .2013; 15(4):1-13.

[9]. Shaftan G W. Indication for operation in abdominal trauma. Am. J. Surg. 1960; 99:657-664

[10]. Hopson W B., Sherman R T., Sanders J W. Stab wounds of the abdomen. Am. Surg. 1966; 32:3:213-218.

[11]. Williams R D and Zollinger R M. Diagnostic and prognostic factors in abdominal trauma. Am.J.Surg 1959; 97:575-578.

[12]. Mc Anena O J, Moore E E, Marx J A. Initial evaluation of the patient with blunt abdominal trauma. Surg Clin North Am 1990; 70:495-515

[13]. Salimi J, Bakhtavar K, Solimani M, Khashayar P, Meysamie A P, Zargar M. Diagnostic accuracy of CT scan in abdominal blunt trauma. Chin J Traumatol. 2009; 12(2):67-70.

[14]. Butela S T, Federle M P, Chang P J, et al. Performance of CT in detection of bowel injury. AJR Am J Roentgenol. 2001; 176(1): $129-135$

[15]. Kim H C, Shin H C, Park S J, et al. Traumatic bowel perforation: analysis of CT findings according to the perforation site and the elapsed time since accident. Clinical Imaging. 2004; 28(5): 334-339.

[16]. Perry JF. Jr. A five year survey of 152 cases of acute abdominal injuries. J Trauma. 1965; 5:53-61.

[17]. Everard F C. Blunt abdominal trauma: a five year analysis of 870 patients requiring celiotomy. Ann. Surg. 1984; 199:467-474.

[18]. Musau P., Jani PG., Owillah FA. (2006) Pattern and outcome of Abdominal injuries AT Kenyatta National Hospital, Nairobi. East Afr Med J.83, 37-43.

[19]. Nyongole O V., Akoko L O., Njile I E., Mwanga A H., Lema LE. Overview of the Pattern of Abdominal Trauma as seen at Muhimbili National Hospital Dar es Salaam, Tanzania. East Cent Afr J Surg 2013; 18:41-48.

[20]. Knudson M M. and Maull K I. Non-operative Management of solid organ injuries-Past, Present and Future. Surg Clin North Am 1999;79:1357-71. 